\title{
Search for the RS model with a small curvature through photon-induced process at the LHC
}

\author{
S. C. İnan ${ }^{1, \mathrm{a}}$, A. V. Kisselev ${ }^{2, \mathrm{~b}}$ \\ ${ }^{1}$ Department of Physics, Cumhuriyet University, 58140 Sivas, Turkey \\ 2 A.A. Logunov Institute for High Energy Physics, NRC "Kurchatov Institute", 142281 Protvino, Russian Federation
}

Received: 4 May 2018 / Accepted: 1 September 2018 / Published online: 10 September 2018

(c) The Author(s) 2018

\begin{abstract}
In this paper, potential of the LHC to explore the phenomenology of the Randall-Sundrum-like scenario with the small curvature for the process $p p \rightarrow p \gamma \gamma p \rightarrow$ $p \mu^{-} \mu^{+} p$ through the subprocess $\gamma \gamma \rightarrow \mu^{-} \mu^{+}$is examined for two forward detector acceptances, $0.0015<\xi<0.5$ and $0.1<\xi<0.5$. This process is known to be one of the most clean channels. The sensitivity bounds on the anomalous model parameters have been found at the $95 \%$ confidence level for various LHC integrated luminosity values.
\end{abstract}

\section{Introduction}

The Standard Model (SM) gives very satisfactory description of high energy physics at an energy scale of electroweak interactions. It is in perfect agreement with current experimental results. However, there are many open equations at the SM. One of this is the hierarchy problem. The extra dimensional models in high energy physics provide possible candidates for this problem. In this respect, these models have drawn attention over the recent years. There are many phenomenology papers of new physics models at the LHC. These searches generally include the usual proton-proton inelastic processes in which the interacted protons dissociate into jets. Due to these jets, such interactions give a very crowded environment. These formed jets create some uncertainties and make it difficult to detect the signals from the new physics which is beyond the SM. However, exclusive production $p p \rightarrow p X p$ provides a very clean environment. These type of processes have been much less examined in the literature. Both interacted protons remain intact, hence they do not dissociate into partons in the exclusive productions. ATLAS and CMS collaborations prepared a physics program of forward physics with extra detectors symmetrically located in a

\footnotetext{
a e-mail: sceminan@cumhuriyet.edu.tr

b e-mail: alexandre.kisselev@ihep.ru
}

distance from the interaction point. These new detectors are equipped with charged particle trackers and they provide to tag intact scattered protons after the collision. Additionally, forward detectors can detect intact outgoing protons in the interval $\xi_{\min }<\xi<\xi_{\max }$ where $\xi$ is the momentum fraction loss of the intact protons $\xi=\left(|E|-\left|E^{\prime}\right|\right) /|E|$ where $E$ and $E^{\prime}$ are the energies of the incoming and intact scattered proton, respectively. These intervals are known as the acceptance of the forward detectors. If these machines are located closer to main detectors, a higher $\xi$ can be created. With applying these new detectors it is possible to obtain high energy photon-photon process with exclusive two particle final states such as leptons or photons. The programs about these detectors were prepared by ATLAS Forward Physics Collaboration (AFP) and CMS-TOTEM Precision Proton Spectrometer (CT-PPS) [1,2]. AFP cover $0.0015<\xi<0.15$, $0.015<\xi<0.15$ detector acceptance ranges. Similarly, CT-PPS has the acceptance ranges of $0.0015<\xi<0.5$, $0.1<\xi<0.5$. Two types of measurements are planned by AFP with high precision: (i) Exploratory physics (anomalous couplings between $\gamma$ and $Z$ or $W$ bosons, exclusive production, etc.), (ii) standard QCD physics (double Pomeron exchange, exclusive production in the jet channel, single diffraction, $\gamma \gamma$ physics, etc.) [3,4]. The CT-PPS experiment main motivations are the investigation of the proton-proton total cross-section, elastic proton-proton interactions, and all of the diffractive processes. Roman Pots detector is used in this experiment. In the forward location, almost all inelastic physical interactions can be detected by the charged particle detectors. A large solid angle unable to cover with the support of the CMS detector. In this way, the detectors can be used for precise studies [5-7]. Due to high energy and high luminosity, this kind of interactions may cause a number of pile-up events. However, these backgrounds enable to be rejected by applied exclusivity conditions, kinematics and timing constraints with use of forward detectors in conjunction with central detectors. Moreover, two 
lepton final states have very small backgrounds in the presence of pile-up events. Because there are no other charged particles on the two lepton interaction vertex. Therefore, final state leptons are highly back-to-back with almost equivalent $p_{t}[8,9]$.

Photon induced reactions were studied by the CDF collaboration $[10,11]$. Obtained results in these experiments are consistent in theoretical expectations with $p \bar{p} \rightarrow p \ell^{-} \ell^{+} \bar{p}$ through the subprocess $\gamma \gamma \rightarrow \ell^{-} \ell^{+}$. At the LHC, the CMS collaboration have recently examined to measurement of the photon-induced reactions through the processes $p p \rightarrow$ $p \gamma \gamma p \rightarrow p \mu^{+} \mu^{-} p, p p \rightarrow p \gamma \gamma p \rightarrow p e^{-} e^{+} p$ from the $\sqrt{s}=7 \mathrm{TeV}[12,13]$. These experiments show that both the number of candidates and the kinematic distributions are in agreement with the expectation for exclusive and semiexclusive $e^{-} e^{+}$via production $\gamma \gamma \rightarrow e^{-} e^{+}$process. Similarly, ATLAS Collaboration reported a measurement of the exclusive $\gamma \gamma \rightarrow l^{-} l^{+}(l=e, \mu)$ cross-section in protonproton collisions at a center-of-mass energy of $7 \mathrm{TeV}$ [14]. In this measurement, it is obtained that when proton absorptive effects due to the finite size of the proton are taken into account, the obtained cross-sections are found to be consistent with the theoretical calculations. Another similar measurement was made by the ATLAS Collaboration at a centerof-mass energy of $8 \mathrm{TeV}$ [15]. In this experiment, the singledifferential cross section was obtained as a function of $m_{\ell^{-} \ell^{+}}$ $(\ell=e, \mu)$. The leptons channel measurements are combined and a total experimental precision of better than $1 \%$ is achieved at low $m_{\ell \ell}$. In the literature, other phenomenological papers based on photon-induced reactions at the LHC for new physics beyond the SM can be found in [16-36].

The forward detectors allow high energy photon-photon interaction as mention above. The photons which are generated by the high energetic protons can be considered as an intense photon beam. These almost-real photons have very low virtuality so that it can be assumed that they are onmass-shell. They radiate off the incoming protons with small angles and low transverse momentum. Intact protons thus deviate slightly from their trajectory along the beam path without being detected by central detectors. Intact protons and $\xi$ are measured by the forward detectors with a very large pseudorapidity. As a result, the final state $X$ is obtained through the process $p p \rightarrow p \gamma \gamma p \rightarrow p X p$ and measured at the main detector. The schematic diagram for this process is shown in Fig. 1. Since, energy loses protons can be detected by the forward detectors, the invariant mass of the central system $W=2 E \sqrt{\xi_{1} \xi_{2}}$ can be known.

The photon-induced reactions can be described by the equivalent photon approximation (EPA) [37,38]. According to this method, quasi-real photons with low vitalities $\left(Q^{2}=-q^{2}\right)$ interact to create final state $\mathrm{X}$ through the subprocess $\gamma \gamma \rightarrow X$. In the EPA framework, emitted quasi-real

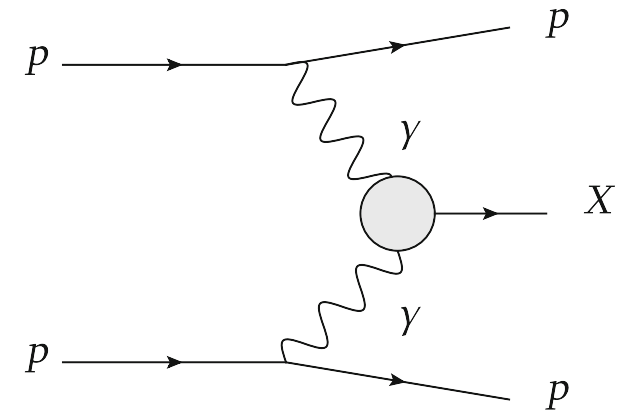

Fig. 1 Schematic diagram for the reaction $p p \rightarrow p \gamma \gamma p \rightarrow p X p$

photons bring a spectrum that is a function of virtuality $Q^{2}$ and the photon energy $E_{\gamma}=\xi E$,

$$
\begin{aligned}
& \frac{d N_{\gamma}}{d E_{\gamma} d Q^{2}}=\frac{\alpha}{\pi} \frac{1}{E_{\gamma} Q^{2}} \\
& \quad \times\left[\left(1-\frac{E_{\gamma}}{E}\right)\left(1-\frac{Q_{\min }^{2}}{Q^{2}}\right) F_{E}+\frac{E_{\gamma}^{2}}{2 E^{2}} F_{M}\right] .
\end{aligned}
$$

Here $m_{p}$ proton mass. In the dipole approximations the other terms given as follows,

$$
\begin{aligned}
& Q_{m i n}^{2}=\frac{m_{p}^{2} E_{\gamma}^{2}}{E\left(E-E_{\gamma}\right)}, \quad F_{E}=\frac{4 m_{p}^{2} G_{E}^{2}+Q^{2} G_{M}^{2}}{4 m_{p}^{2}+Q^{2}}, \\
& G_{E}^{2}=\frac{G_{M}^{2}}{\mu_{p}^{2}}=\left(1+\frac{Q^{2}}{Q_{0}^{2}}\right)^{-4}, \quad F_{M}=G_{M}^{2}, \\
& Q_{0}^{2}=0.71 \mathrm{GeV}^{2} .
\end{aligned}
$$

where, $\mu_{p}^{2}=7.78$ is the square of the magnetic moment of the proton, $F_{E}$ and $F_{M}$ are the relative the electric and magnetic form factors of the proton, respectively. In the photonphoton collisions, luminosity spectrum $\frac{d L^{\gamma \gamma \gamma}}{d W}$ can be found with using EPA as follows,

$$
\begin{aligned}
\frac{d L^{\gamma \gamma}}{d W}= & \int_{Q_{1, \min }^{2}}^{Q_{\max }^{2}} d Q_{1}^{2} \int_{Q_{2, \min }^{2}}^{Q_{\max }^{2}} d Q_{2}^{2} \int_{y_{\min }}^{y_{\max }} d y \frac{W}{2 y} \\
& \times f_{1}\left(\frac{W^{2}}{4 y}, Q_{1}^{2}\right) f_{2}\left(y, Q_{2}^{2}\right)
\end{aligned}
$$

with $y_{\min }=\max \left(W^{2} /\left(4 \xi_{\max } E\right), \xi_{\min } E\right), y_{\max }=\xi_{\max } E$, $f=\frac{d N}{d E_{\gamma} d Q^{2}}$. Here, $Q_{\text {max }}^{2}=2 \mathrm{GeV}^{2}$ is taken in the above equation since the contribution of higher virtualities more than this value is negligible. The cross section for the $p p \rightarrow p \gamma \gamma p \rightarrow p X p$ can be derived by integrating selected subprocess $\gamma \gamma \rightarrow X$ cross section over the photon spectrum,

$d \sigma=\int \frac{d L^{\gamma \gamma}}{d W} d \hat{\sigma}_{\gamma \gamma \rightarrow X}(W) d W$.

In this paper, we have investigated the RS-like scenario with the small curvature (the details is given in the next section) for the process $p p \rightarrow p \gamma \gamma p \rightarrow p \mu^{-} \mu^{+} p$ through the 


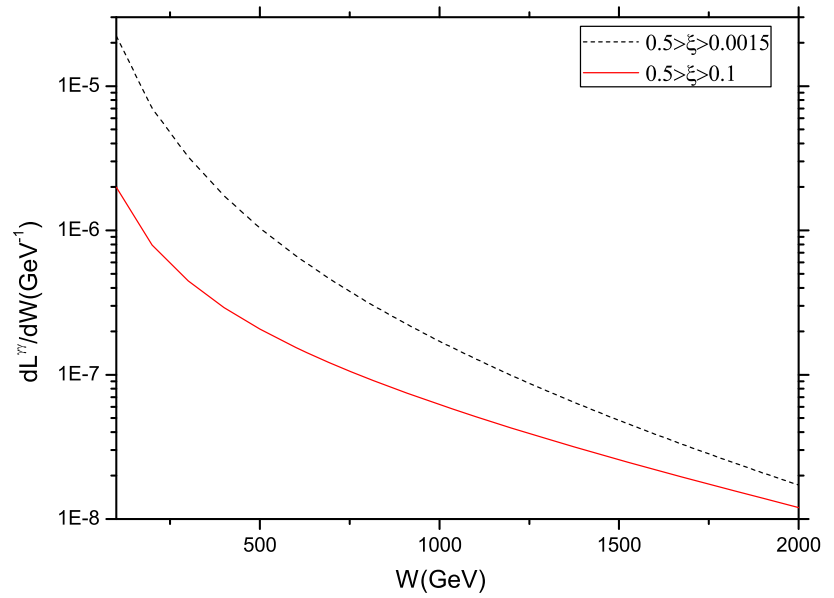

Fig. 2 Effective $\gamma \gamma$ luminosity as a function of the invariant mass of the two photon system. Figure shows the effective luminosity for two forward detector acceptances: $0.0015<\xi<0.5$ and $0.1<\xi<0.5$

subprocess $\gamma \gamma \rightarrow \mu^{-} \mu^{+}$. Because of the main contribution comes from the high energy region, we have made this calculation for two acceptance ranges $0.0015<\xi<0.5$ and $0.1<\xi<0.5$. Figure 2 shows the behavior of the $\gamma \gamma$ luminosity spectrum as a function of the $W$ for these two forward acceptance ranges.

\section{RSSC model of warped extra dimension with small curvature}

The Randall-Sundrum model with two branes (RS1 model [39]) was proposed as an alternative to the scenario with large flat extra dimensions (ADD model [40-42]). The RS1 model is described by the following background warped metric

$d s^{2}=e^{-2 \sigma(y)} \eta_{\mu \nu} d x^{\mu} d x^{\nu}-d y^{2}$,

where $\eta_{\mu \nu}$ is the Minkowski tensor with the signature $(+,-,-,-)$, and $y$ is an extra coordinate. The periodicity condition $y=y+2 \pi r_{c}$ is imposed, and the points $\left(x_{\mu}, y\right)$ and $\left(x_{\mu},-y\right)$ are identified. Thus, we have a model of gravity in a slice of the $\mathrm{AdS}_{5}$ space-time compactified to the orbifold $S^{1} / Z_{2}$. The orbifold has two fixed points, $y=0$ and $y=\pi r_{c}$. It is assumed that there are two branes located at these points (called Planck and TeV brane, respectively). All the SM fields are confined to the TeV brane.

The classical action of the RS1 model is given by [39]

$$
\begin{aligned}
S= & \int d^{4} x \int_{-\pi r_{c}}^{\pi r_{c}} d y \sqrt{G}\left(2 \bar{M}_{5}^{3} \mathcal{R}-\Lambda\right) \\
& +\int d^{4} x \sqrt{\left|g^{(1)}\right|}\left(\mathcal{L}_{1}-\Lambda_{1}\right)+\int d^{4} x \sqrt{\left|g^{(2)}\right|}\left(\mathcal{L}_{2}-\Lambda_{2}\right),
\end{aligned}
$$

where $G_{M N}(x, y)$ is the 5-dimensional metric, with $M, N=$ $0,1,2,3,4, \mu=0,1,2,3$. The quantities

$g_{\mu \nu}^{(1)}(x)=G_{\mu \nu}(x, y=0), \quad g_{\mu \nu}^{(2)}(x)=G_{\mu \nu}\left(x, y=\pi r_{c}\right)$

are induced metrics on the branes, $\mathcal{L}_{1}$ and $\mathcal{L}_{2}$ are brane Lagrangians, $G=\operatorname{det}\left(G_{M N}\right), g^{(i)}=\operatorname{det}\left(g_{\mu \nu}^{(i)}\right) . \bar{M}_{5}$ is a reduced 5-dimensional Planck scale, $M_{5} /(2 \pi)^{1 / 3}$, where $M_{5}$ is a fundamental gravity scale. The quantity $\Lambda$ is a 5dimensional cosmological constant, $\Lambda_{1,2}$ are brane tensions.

The function $\sigma(y)$ in (6) was obtained in [39] to be

$\sigma_{\mathrm{RS}}(y)=\kappa|y|$

where $\kappa$ is a parameter with a dimension of mass. It defines the curvature of the 5-dimensional space-time. Recently, a generalization of the warp factor $\sigma(y)$ war derived in [43]

$$
\begin{aligned}
\sigma(y)= & \frac{\kappa r_{c}}{2}\left[\left|\operatorname{Arccos}\left(\cos \frac{y}{r_{c}}\right)\right|-\left|\pi-\operatorname{Arccos}\left(\cos \frac{y}{r_{c}}\right)\right|\right] \\
& +\frac{\pi|\kappa| r_{c}}{2}-C
\end{aligned}
$$

where $C$ is $y$-independent quantity, with the fine tuning relations

$\Lambda=-24 \bar{M}_{5}^{3} \kappa^{2}, \quad \Lambda_{1}=-\Lambda_{2}=24 \bar{M}_{5}^{3} \kappa$.

Here $\operatorname{Arccos}(z)$ is a principal value of the multivalued inverse trigonometric function $\arccos (z)$. This generalized solution (i) obeys the orbifold symmetry $y \rightarrow-y$; (ii) makes the jumps of $\sigma^{\prime}(y)$ on both branes; (iii) has the explicit symmetry with respect to the branes [43].

By taking $C=0$ in (10), we get the RS1 model (9), while putting $C=\pi \kappa r_{c}$, we come to the RS-like scenario with the small curvature of the space-time (RSSC model [44-46]). What are main features of the RSSC model in comparison with those of the RS1 model?

The interactions of the Kaluza-Klein (KK) gravitons $h_{\mu \nu}^{(n)}$ with the SM fields on the TeV brane are given by the effective Lagrangian density

$$
\begin{aligned}
\mathcal{L}_{\mathrm{int}}= & -\frac{1}{\bar{M}_{\mathrm{Pl}}} h_{\mu \nu}^{(0)}(x) T_{\alpha \beta}(x) \eta^{\mu \alpha} \eta^{\nu \beta} \\
& -\frac{1}{\Lambda_{\pi}} \sum_{n=1}^{\infty} h_{\mu \nu}^{(n)}(x) T_{\alpha \beta}(x) \eta^{\mu \alpha} \eta^{\nu \beta},
\end{aligned}
$$

were $\bar{M}_{\mathrm{Pl}}=M_{\mathrm{Pl}} / \sqrt{8 \pi}$ is the reduced Planck mass, $T^{\mu \nu}(x)$ is the energy-momentum tensor of the SM fields. The coupling constant is equal to

$\Lambda_{\pi}=\bar{M}_{5} \sqrt{\frac{\bar{M}_{5}}{\kappa}}$.

The hierarchy relation looks like

$$
\bar{M}_{\mathrm{Pl}}^{2}=\left.\frac{\bar{M}_{5}}{\kappa}\left[e^{2 \pi \kappa r_{c}}-1\right]\right|_{\kappa \pi r_{c} \gg 1}=\frac{\bar{M}_{5}}{\kappa} e^{2 \pi \kappa r_{c}} .
$$


The masses of the KK gravitons are proportional to the curvature parameter $\kappa$ [45]

$m_{n}=x_{n} \kappa, \quad n=1,2, \ldots$,

where $x_{n}$ are zeros of the Bessel function $J_{1}(x)$. If we put $\kappa \ll \bar{M}_{5} \sim 1 \mathrm{TeV}$, the mass splitting will be small, $\Delta m \simeq$ $\pi \kappa$, and we come to an almost continuous mass spectrum, similar to the mass spectrum of the ADD model [40]. This is in contrast to the RS1 model, in which the gravitons are heavy resonances with masses above one-few $\mathrm{TeV}$.

It is worth to stress that the RSSC model first proposed in [44] and after that developed in Refs. [43,45,46] differs significantly from the 5-dimensional space-time scenario with one flat extra dimension (ED) even for small value of $\kappa$. To demonstrate this, consider the hierarchy relation for the ADD model with $n$ EDs of the size $r_{c}$ [40-42]

$\bar{M}_{\mathrm{Pl}}^{2}=\left(2 \pi r_{c}\right)^{n} M_{D}^{2+n}$

where $M_{D}$ is a fundamental gravity scale in $D=4+n$ dimensions. For $n=1$ this equation is a particular case of the hierarchy relation (14) in the limit $2 \kappa \pi r_{c} \ll 1$. However, the condition $2 \kappa \pi r_{c} \ll 1$ means that [46]

$\frac{\bar{M}_{5}}{\kappa} \gg\left(\frac{\bar{M}_{\mathrm{Pl}}}{\bar{M}_{5}}\right)^{2}$.

Inequality (17) is satisfied if only $\kappa \ll 10^{-22} \mathrm{eV}$ for $\bar{M}_{5}=$ $1 \mathrm{TeV}$. Thus, we conclude that the RSSC model cannot be considered as a simple "distortion" of the ADD model with one ED. The smallness of $\kappa$ means that $\kappa \ll \bar{M}_{5}$, that is, $\kappa$ is very small in comparison with the curvature in the original RS model [39] in which $\kappa \sim M_{\mathrm{Pl}}$.

Let us calculate the scattering amplitude for the subprocess $\gamma \gamma \rightarrow l^{-} l^{+}$by adding $s$-channel KK graviton exchange to the SM electromagnetic contribution, which is defined as

$$
\begin{aligned}
M_{K K}= & \frac{1}{2 \Lambda_{\pi}^{2}} \sum_{n}\left[\bar{u}\left(p_{1}\right) \Gamma_{2}^{\mu v} v\left(p_{2}\right)\right. \\
& \left.\times \frac{B_{\mu \nu \alpha \beta}}{\hat{s}-m_{n}^{2}} \Gamma_{1}^{\alpha \beta \rho \sigma} e_{\rho}\left(k_{1}\right) e_{\sigma}\left(k_{2}\right)\right],
\end{aligned}
$$

where $k_{1}, k_{2}, p_{1}, p_{2}$ and $e_{\rho}\left(k_{i}\right)$ are incoming photon, outgoing lepton momenta and polarization vectors of photons. The coherent sum is over KK modes. Vertex functions $\Gamma_{1}^{\alpha \beta \rho \sigma}$ for $K K-\gamma \gamma$ and $\Gamma_{2}^{\mu \nu}$ for $K K-\ell \ell$ are given below

$$
\begin{aligned}
\Gamma_{1}^{\alpha \beta \rho \sigma} & =-\frac{i}{2}\left[\left(k_{1} \cdot k_{2}\right) C^{\alpha \beta \rho \sigma}+D^{\alpha \beta \rho \sigma}\right], \\
\Gamma_{2}^{\mu \nu} & =-\frac{i}{8}\left[\gamma^{\mu}\left(p_{1}^{\nu}-p_{2}^{\nu}\right)+\gamma^{\nu}\left(p_{1}^{\mu}-p_{2}^{\mu}\right)\right] .
\end{aligned}
$$

Explicit forms of the tensors $C^{\alpha \beta \rho \sigma}$ and $D^{\alpha \beta \rho \sigma}$ are given by Eqs. (A.1) and (A.2) in Appendix A, while $B_{\mu \nu \alpha \beta}$ is a tensor part of the graviton propagator

$B_{\mu \nu \alpha \beta}=\eta_{\mu \alpha} \eta_{\nu \beta}+\eta_{\mu \beta} \eta_{\nu \alpha}-\frac{2}{3} \eta_{\mu \nu} \eta_{\alpha \beta}$

The total amplitude squared consists of electromagnetic, KK and interference parts [47]

$|M|^{2}=\left|M_{\mathrm{em}}\right|^{2}+\left|M_{\mathrm{KK}}\right|^{2}+\left|M_{\mathrm{int}}\right|^{2}$,

where

$$
\begin{aligned}
\left|M_{\mathrm{em}}\right|^{2} & =-2 g_{e}^{4}\left[\frac{\hat{s}+\hat{t}}{\hat{t}}+\frac{\hat{t}}{\hat{s}+\hat{t}}\right], \\
\left|M_{\mathrm{KK}}\right|^{2} & =\frac{1}{4}|S(\hat{s})|^{2}\left[-\frac{\hat{t}}{8}\left(\hat{s}^{3}+2 \hat{t}^{3}+3 \hat{t} \hat{s}^{2}+4 \hat{t}^{2} \hat{s}\right)\right], \\
\left|M_{\mathrm{int}}\right|^{2} & =-\frac{1}{4} g_{e}^{2} \operatorname{Re} S(\hat{s})\left[\hat{s}^{2}+2 \hat{t}^{2}+2 \hat{s} \hat{t}\right] .
\end{aligned}
$$

Here $\hat{s}, \hat{t}$ are Mandelstam variables of the subprocess $\gamma \gamma \rightarrow$ $l^{-} l^{+}$, and $g_{e}^{2}=4 \pi \alpha_{\mathrm{em}}$.

The $s$-channel contribution of the KK gravitons in (24) and (25) is given by the expression

$\mathcal{S}(s)=\frac{1}{\Lambda_{\pi}^{2}} \sum_{n=1}^{\infty} \frac{1}{s-m_{n}^{2}+i m_{n} \Gamma_{n}}$,

were $\Gamma_{n}$ denotes the total width of the KK graviton with the mass $m_{n}$. The sum (26) has been calculated in Ref. [46]

$\mathcal{S}(s)=-\frac{1}{4 \bar{M}_{5}^{3} \sqrt{s}} \frac{\sin (2 A)+i \sinh (2 \varepsilon)}{\cos ^{2} A+\sinh ^{2} \varepsilon}$,

where

$A=\frac{\sqrt{s}}{\kappa}, \quad \varepsilon=0.045\left(\frac{\sqrt{s}}{\bar{M}_{5}}\right)^{3}$.

The virtual graviton exchange should lead to deviations from the SM predictions both in a magnitude of the cross sections and in an angular distribution of the final leptons because of the spin-2 nature of the gravitons.

\section{Numerical analysis}

The KK graviton exchange studied in the previous section should lead to deviations from the SM in magnitudes both of differential cross sections and of total cross sections for the photon-induced process $p p \rightarrow p \mu^{+} \mu^{-} p$ at the LHC which goes via subprocess $\gamma \gamma \rightarrow \mu^{+} \mu^{-}$. Our goal is to calculate the dependence of these deviations on the parameters of the RSSC model.

As it was mentioned in Sect. 1, the process can be detected by using forward detectors CN-PPS (CMS-TOTEM Collaboration) and AFP (ATLAS Collaboration). In what follows, 
we will consider two acceptance regions of the forward detectors, $0.0015<\xi<0.5$ and $0.1<\xi<0.5$.

Before describing our numerical results, it is worth to make a few remarks about using the cut imposed on the muon rapidities during our calculations. In the c.m.s. of the colliding protons, two-muon system $X$ moves with the rapidity

$\eta_{X}=\frac{1}{2} \ln \frac{E_{1}}{E_{2}}=\frac{1}{2} \ln \frac{\xi_{1}}{\xi_{2}}$,

where $E_{1,2}=\xi_{1,2} E$ are the energies of the photons, $4 E_{1} E_{2}=\hat{s}=W^{2}, 2 E=\sqrt{s}$ is the invariant energy of the $p p$ collision. The rapidities of the muons in the c.m.s. of two photons $\left(=\right.$ c.m.s of the system $X$ ) are equal to $\eta_{\gamma \gamma}$ and $-\eta_{\gamma \gamma}$, respectively. $\eta_{\gamma \gamma}$ depends on the scattering angle $\theta_{\gamma \gamma}$ in the c.m.s. of the subprocess $\gamma \gamma \rightarrow \mu^{-} \mu^{+}$

$\eta_{\gamma \gamma}=\ln \left(\cot \frac{\theta_{\gamma \gamma}}{2}\right)=\ln \frac{W+\sqrt{W^{2}-4 p_{t}^{2}}}{2 p_{t}}$.

Correspondingly, the muon rapidities in the c.m.s. of the colliding protons are equal to

$\eta_{p p}^{(1)}=\eta_{\gamma \gamma}+\eta_{X}$

$\eta_{p p}^{(2)}=-\eta_{\gamma \gamma}+\eta_{X}$

It is clear that the rapidity cuts $\left|\eta_{p p}^{(1,2)}\right|<\eta_{\max }$ are equivalent to the inequality

$\eta_{\gamma \gamma}<\eta_{\max }-\left|\eta_{X}\right|$

In our case $\eta_{\max }=2.4$. Taking into account the cut imposed on the transverse momenta of the muons, $\left|p_{t}\right|>p_{t \text {, min }}$, one can transform inequality (32) into the bound on the scattering angle of the muons in the c.m.s of two photons

$\left|\cos \theta_{\gamma \gamma}\right|<(\cos \theta)_{\max }$

where

$$
\begin{aligned}
& (\cos \theta)_{\max } \\
& = \begin{cases}0, & \text { if }\left|\eta_{X}\right| \geqslant \eta_{\max }, \\
\min \left[\sqrt{1-\left(\frac{2 p_{\mathrm{t} \min }}{W}\right)^{2}}, \tanh \left(\eta_{\max }\right)\right], & \text { if }\left|\eta_{X}\right|<\eta_{\max } .\end{cases}
\end{aligned}
$$

It is convenient to use the rapidity cut in such a form (34). The reason is that the electromagnetic part of the matrix element $|M|^{2}$ (23) depends only on $\cos \theta_{\gamma \gamma}$, while other two terms in $|M|^{2},(24)$ and (25), are functions of the variable $\cos \theta_{\gamma \gamma}$ multiplied by functions of $\hat{s}$. Thus, the corresponding integrals ( $a=\mathrm{em}, \mathrm{KK}$, int)

$$
\int_{-(\cos \theta)_{\max }}^{(\cos \theta)_{\max }} \frac{d \sigma_{a}}{d \cos \theta_{\gamma \gamma}} d \cos \theta_{\gamma \gamma}
$$

can be calculated analytically.
The inequality $\left|\eta_{X}\right|<\eta_{\max }$ means that allowable values of the muon transverse momenta are bounded from below

$p_{t}>p_{t, 0}=\frac{W_{\min }}{\cosh \left(\eta_{\max }\right)}$,

where $W_{\min }=2 \xi_{\min } E$. Thus, for fixed $p_{t}>p_{t, 0}$, the following kinematical bounds take place

$\max \left(W_{\min }, 2 p_{t}\right) \leqslant W \leqslant W_{\max }$,

where $W_{\max }=2 \xi_{\max } E$.

The results of our numerical calculations of the differential cross sections $d \sigma / d p_{t}$ as a function of the transverse momenta of the muons are presented in Figs. 3, 4 and 5. As one can see, $d \sigma / d p_{t}$ exceeds the SM contribution $d \sigma_{\mathrm{SM}} / d p_{t}$ for $p_{t}>500 \mathrm{GeV}$, and the difference between $d \sigma / d p_{t}$ and $d \sigma_{\mathrm{SM}} / d p_{t}$ increases as $p_{t}$ grows. The effect is more pronounced for small values of $\bar{M}_{5}$, for which $d \sigma / d p_{t}$ becomes dominant already for $p_{t} \gtrsim 500-600 \mathrm{GeV}$.

Some comments should be made on a possible $\bar{M}_{5}$ dependence of $d \sigma / d p_{t}$. One may naively expect from Eq. (27) that the KK contribution to the differential cross section should be

$\left.\frac{d \sigma_{\mathrm{KK}}}{d p_{t}}\right|_{\text {naive }} \sim \frac{1}{\bar{M}_{5}^{6}}$.

Nevertheless, the results of our numerical calculations shows that

$\frac{d \sigma_{\mathrm{KK}}}{d p_{t}} \sim \frac{1}{\bar{M}_{5}^{3}}$,

at small and moderate values of $p_{t}$. As a result, the differential cross section $d \sigma / d p_{t}$ follows this $\bar{M}_{5}$-dependence. At large $p_{t}$ the $\bar{M}_{5}$-dependence tends to the form $\bar{M}_{5}^{-6}$. It can be explained as follows [48,49]. In the RSSC model the invariant part of the scattering amplitude (27) is a sum of rather sharp resonances whose widths are proportional to $\kappa^{4} / \bar{M}_{5}^{3}$. The contribution of one resonance can be estimated as $[48,49]$

$\left.\frac{d \sigma_{\mathrm{KK}}}{d p_{t}}\right|_{\text {one res }} \sim \frac{\kappa}{\bar{M}_{5}^{3} W}$.

Taking into account that the total number of the graviton resonances which contribute to the differential cross section $\sim W / \kappa$, we come to Eq. (39).

From Eq. (5) with using dimensionless parameter $z=$ $\sqrt{W^{2} / 4 p_{t}^{2}-1}$ instead of variable $W$, the SM differential cross section can be obtained analytically as follows

$$
\begin{aligned}
\frac{d \sigma_{\mathrm{SM}}}{d p_{t}}= & \frac{g e^{4}}{16 \pi} \frac{1}{p_{t}^{3}} \int_{z_{\min }}^{z_{\max }} d z \frac{2 z^{2}+1}{\left(z^{2}+1\right)^{5 / 2}} \int \frac{d \xi_{2}}{\xi_{2}} \int \frac{d Q_{1}^{2}}{Q_{1}^{2}} \int \frac{d Q_{2}^{2}}{Q_{2}^{2}} \\
& \times \tilde{f}_{1}\left(\frac{W^{2}(z)}{4 E^{2} \xi_{2}}\right) \tilde{f}_{2}\left(\xi_{2}, Q_{2}^{2}\right),
\end{aligned}
$$



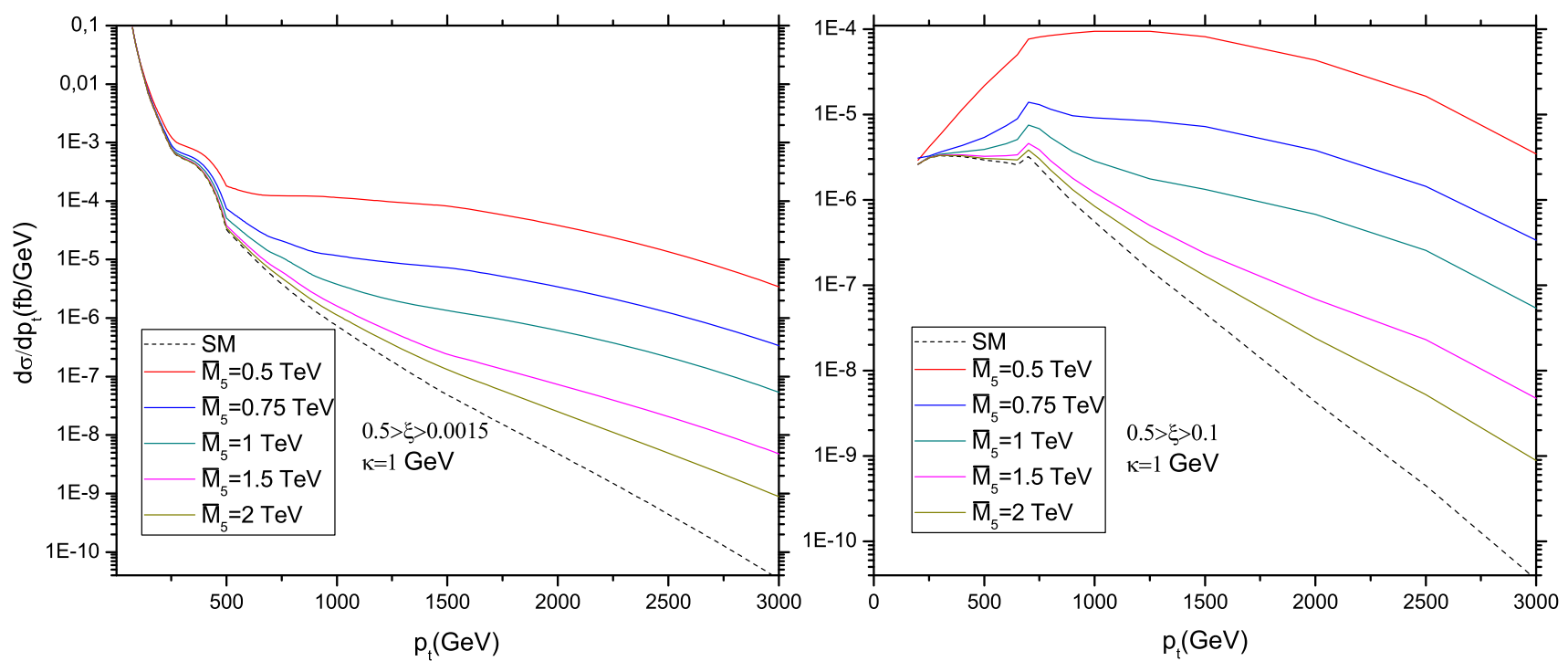

Fig. 3 The differential cross section for the process $p p \rightarrow p \mu^{+} \mu^{-} p$ as a function of the transverse momenta of the final muons for $\kappa=1 \mathrm{GeV}$ and two acceptance regions. The cut on the muon rapidities, $|\eta|<2.4$, is imposed. Left panel: $0.0015<\xi<0.5$. Right panel: $0.1<\xi<0.5$. Here and below the dotted line denotes the SM contribution
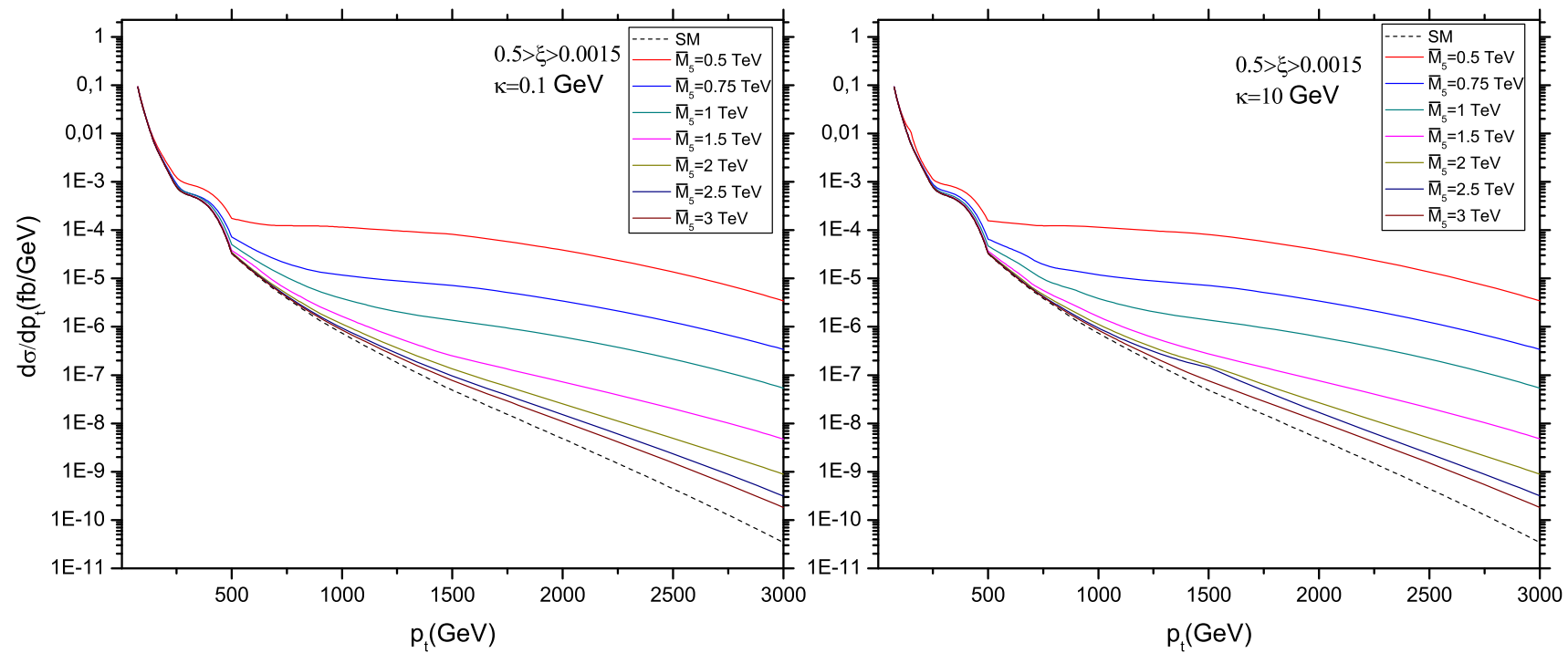

Fig. 4 The differential cross section for the process $p p \rightarrow p \mu^{+} \mu^{-} p$ as a function of the transverse momenta of the final muons for the acceptance region $0.0015<\xi<0.5$. Left panel: $\kappa=0.1 \mathrm{GeV}$. Right panel: $\kappa=10 \mathrm{GeV}$

where

$\tilde{f}_{i}\left(\xi, Q^{2}\right)=\xi_{i} Q_{i}^{2} \frac{d N_{\gamma}\left(\xi, Q^{2}\right)}{\xi_{i} d Q_{i}^{2}}$

is a reduced dimensionless photon distribution $(i=1,2)$ and

$z_{\min }=\sqrt{\frac{W_{\min }^{2}}{4 p_{t}^{2}}-1}, \quad z_{\max }=\sqrt{\frac{W_{\max }^{2}}{4 p_{t}^{2}}-1}$,

$W_{\min }=\max \left(\xi_{\min } 2 E, 2 p_{t}\right), \quad W_{\max }=\xi_{\max } 2 E$.
The $p_{t}$-dependence of the integrand in (41) results in (after integrations in all variables) both the small dip (around the $600 \mathrm{GeV}$ ) and two maxima (first is around the $300 \mathrm{GeV}$ and second is around the $700 \mathrm{GeV}$ ) for the SM contribution as seen from Fig. 5.

Figure 5 demonstrates to us that the differential cross section is almost independent of the curvature parameter $\kappa$, with the exception of its weak dependence on $\kappa$ around the point $p_{t}=750 \mathrm{GeV}$. Thus, we can put limits on the fundamental gravity scale $\bar{M}_{5}$ regardless of the parameter $\kappa$ (provided $\kappa \ll \bar{M}_{5}$ is satisfied). This is in contrast to the RS1 model 


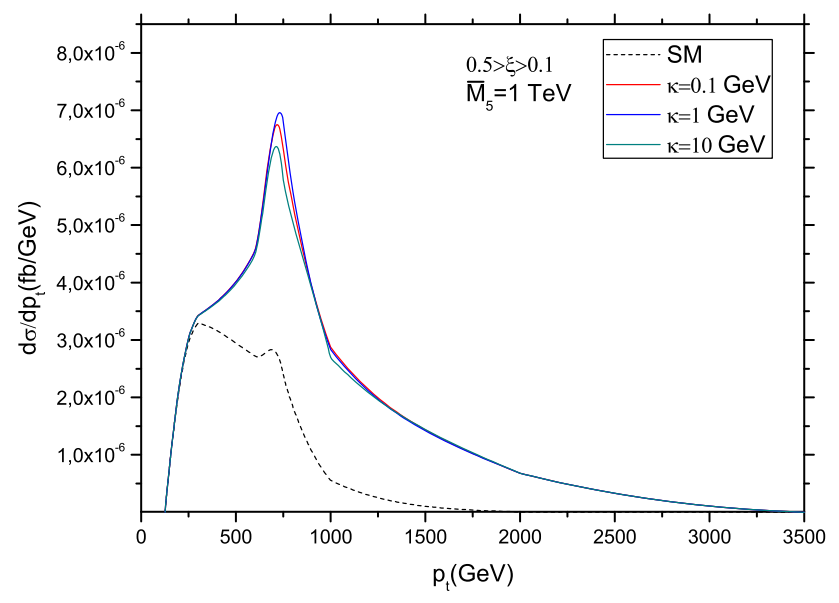

Fig. 5 The differential cross section for the process $p p \rightarrow p \mu^{+} \mu^{-} p$ as a function of the transverse momenta of the final muons for the acceptance region $0.1<\xi<0.5$ for different values of $\kappa$

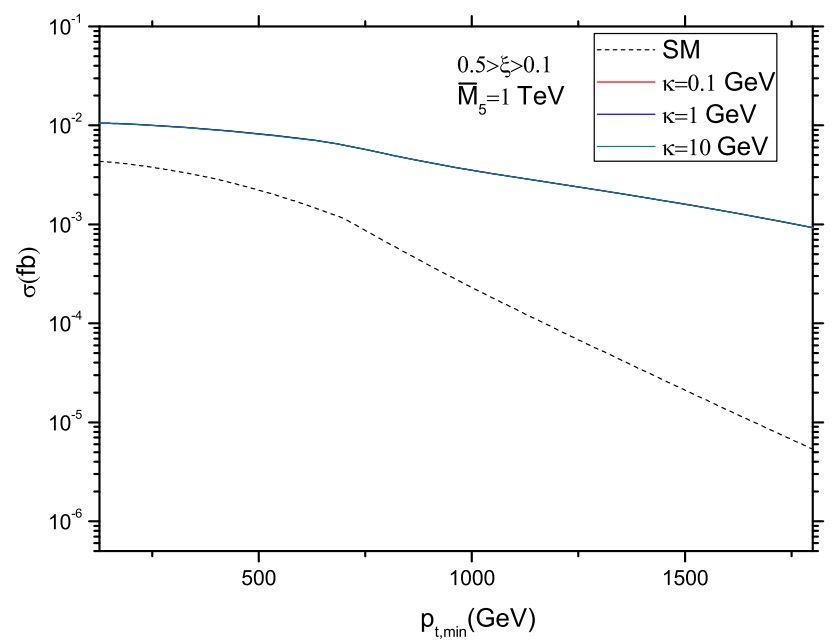

Fig. 6 The total cross section for the process $p p \rightarrow p \mu^{+} \mu^{-} p$ as a function of the minimal transverse momenta of the final muons $p_{t, \min }$ for the acceptance region $0.1<\xi<0.5$ for different values of $\kappa$

[39] in which all cross sections depend substantially on the ratio $\beta=\kappa / M_{\mathrm{Pl}}$.

The next two Figs. 6 and 7 shows us the total cross sections with and without KK graviton exchange versus the minimal transverse momentum of the final muons $p_{t, \min }$. The comparison with the pure SM predictions is also given. From all said above it is not surprising that the quantity $\sigma\left(p_{t}>p_{t, \min }\right)$ does not depend on $\kappa$ (see Fig. 6). For both acceptance regions, its deviation from the $\mathrm{SM}$ gets higher as $p_{t, \text { min }}$ grows (see Fig. 7). When the two figures are compared, we can see that the $0.1<\xi<0.5$ case has almost the same behaviour as the case $0.0015<\xi<0.5$ with $p_{t, \text { min }} \sim 500 \mathrm{GeV}$. Moreover, for the $0.1<\xi<0.5$ acceptance region, as the $p_{t, \text { min }}$ changes, the cross sections almost do not change if $0<p_{t, \min }<500$. Therefore, it can be said that a high value of $\xi_{\min }$ mimics a high value of $p_{t, \min }$.
In this motivation, we have obtained the limits on the $\bar{M}_{5}$ for two cases: $0.0015<\xi<0.5$ for $p_{t}>500 \mathrm{GeV}$ and $0.1<\xi<0.5$ for $p_{t}>30 \mathrm{GeV}$. In sensitivity analysis, the likelihood method are used. In this method, it is assumed that the entire number of background events in every signal location respects the normal distribution with a fractional uncertainty. The statistical significance is obtained as follows [50],

$S S=\sqrt{2[(S+B) \ln (1+S / B)-S]}$,

where $S, B$ are the signal and background events number, respectively. Here, we have assumed that the uncertainty of the background is negligible. Using our results on the cross sections, we have calculated 95\% CL bounds on $\bar{M}_{5}$ for two acceptance regions, $0.0015<\xi<0.5$ with $p_{t}>500 \mathrm{GeV}$ and $0.1<\xi<0.5$ with $p_{t}>30 \mathrm{GeV}$ with using Eq. (45). The bounds are presented in Fig. 8 as a function of the integrated LHC luminosity. Here, we assumed that the $\kappa=1$ $\mathrm{GeV}$. Note that the cut $|\eta|<2.4$ was imposed on the rapidities on the final muons. The results are presented in Fig. 8 which have nearly the same behavior for the two acceptances regions. It can be see that $95 \%$ CL sensitivity of $\bar{M}_{5}$ is about $1430 \mathrm{GeV}$ for the $0.0015<\xi<0.5$ and $1265 \mathrm{GeV}$ for the $0.1<\xi<0.5$ when the LHC integrated luminosity values are equal to $3000 \mathrm{fb}^{-1}$.

Our bounds on the 5-dimensional gravity scale $\bar{M}_{5}$ are rather low in comparison with the LHC bounds on $D$ dimensional scale in the ADD model $M_{D}$. In this regard, we would like to emphasize the following. The LHC bounds on $M_{D}$ cannot be directly applied to the gravity scale of the RSSC model. As was shown above (see comments after Eq. (15)), this model cannot be regarded as a small distortion of the ADD model. Moreover, in the ADD model the number of EDs should be $n \geqslant 2$, while in the RSSC scenario we deal with one extra dimension, $n=1$. As for the original RS model, it has $\bar{M}_{5} \sim \kappa \sim M_{\mathrm{Pl}}$, and bounds are put on the ratio $\kappa / \bar{M}_{5}$ and mass of the lightest KK graviton $m_{1}$. We consider the diphoton production in the photon induced process at the LHC as another means of seeing effects of low $M_{5}$ in the RS-like scenario with the small curvature.

A framework that allows a self-consistent description of quantum gravity is string theory [51]. In the presence of extra dimensions, the string scale $M_{S}$ and the fundamental Planck scale $M_{P}$ could be as low as $\sim \mathrm{TeV}$ [40-42]. The TeV-gravity theory has four new types of particles (higher-dimensional gravitons, low-lying string excitations, string balls (SB) [52] and black holes (BH) [53-56]) and three associated mass scales ( $M_{P}, M_{s}$, and $M_{s} / g_{s}^{2}$, where $g_{s}$ is the string coupling).

If $g_{s} \ll 1$ (perturbative string theory), there is a separation between these scales. In the type I string theory with Dbranes the scales $M_{P}$ and $M_{S}$ are related with each other [42]. Taking into account that in some respect (small curvature of the space-time, almost continuous spectrum of massive 

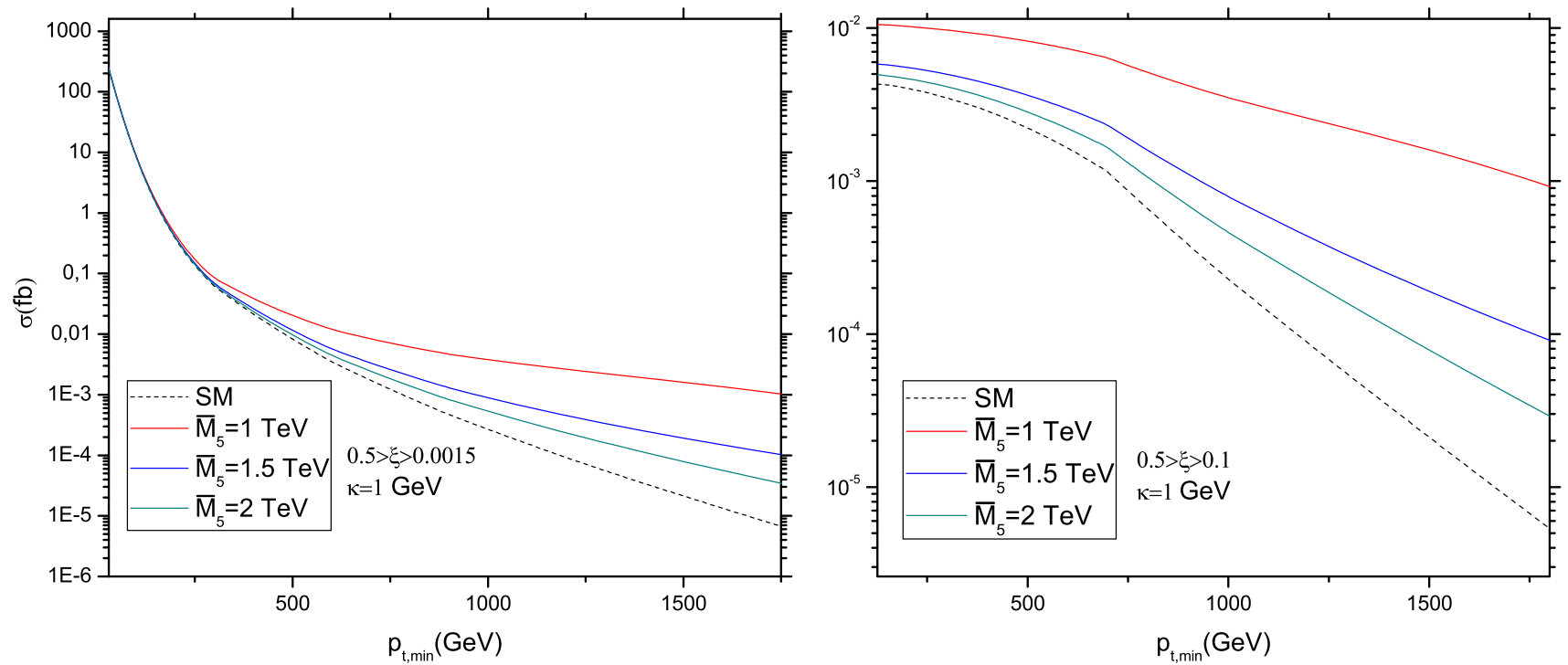

Fig. 7 The total cross section for the process $p p \rightarrow p \mu^{+} \mu^{-} p$ as a function of the minimal transverse momenta of the final muons $p_{t, \text { min }}$ for different values of $\bar{M}_{5}$. Left panel: $0.0015<\xi<0.5$. Right panel: $0.1<\xi<0.5$
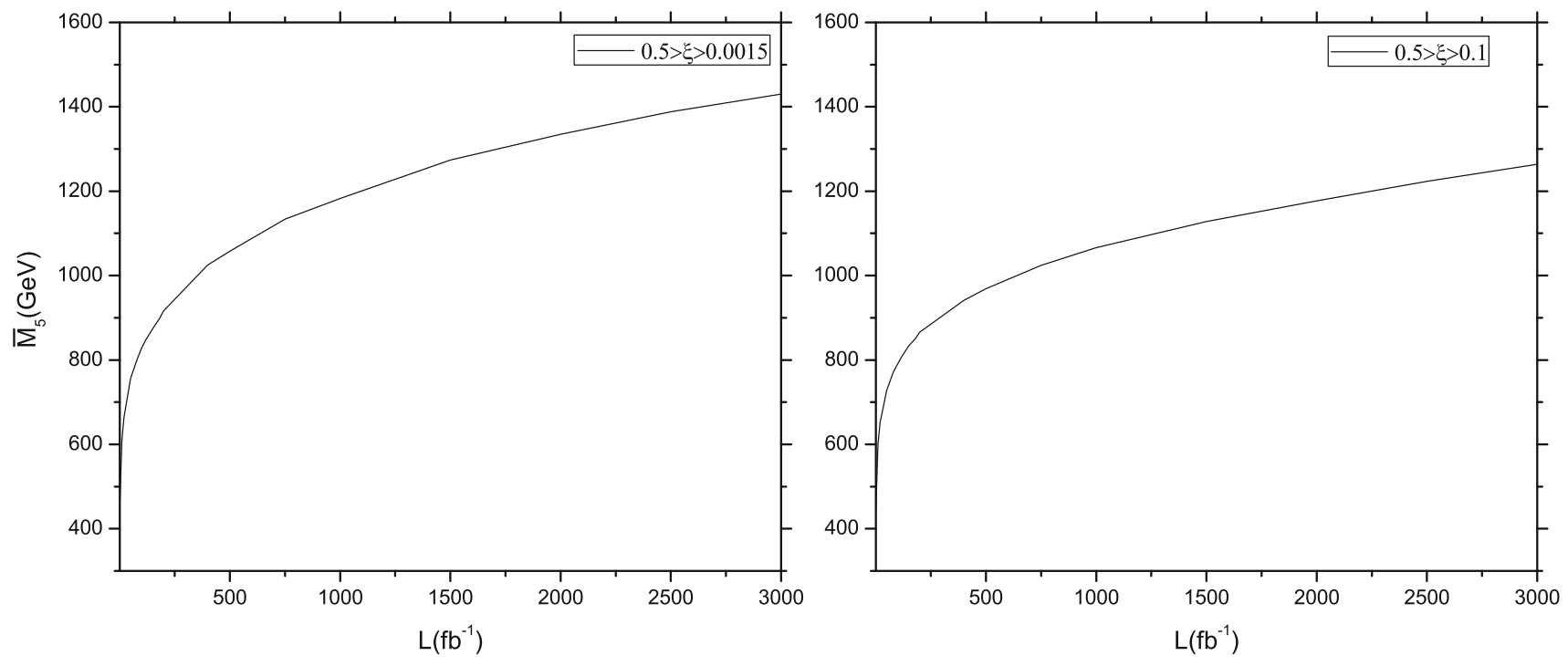

Fig. 8 The $95 \%$ CL search limits for the reduced 5-dimensional gravity scale $\bar{M}_{5}$ as a function of the integrated LHC luminosity for $0.0015>$ $\xi>0.5$ with $p_{t}>500 \mathrm{GeV}$ and $0.1<\xi<0.5$ with $p_{t}>30 \mathrm{GeV}$. The rapidity cut of 2.4 on the muon rapidities are imposed

gravitons) the RSSC model is similar to the ADD model with one extra dimension, we have

$\frac{M_{s}}{M_{P}}=(4 \pi \alpha)^{2 / 3}$,

where $\alpha=g^{2} /(4 \pi)$ is the gauge coupling, and the proportionality constant follows from the convention of Ref. [57]. For instance, we find that $M_{s} / M_{P}$ is equal to $0.25(1.16)$ for $\alpha=0.01(0.1)$. Typically,

$$
M_{s}<M_{P}<\frac{M_{s}}{g_{s}^{2}}
$$

$M_{s} \ll M_{\mathrm{SB}} \ll \frac{M_{s}}{g_{s}^{2}}$,

$\frac{M_{s}}{g_{s}^{2}} \ll M_{\mathrm{BH}}$,

where $M_{\mathrm{SB}}$ is the string ball mass, while $M_{\mathrm{BH}}$ is the mass of the black hole.

If $g_{s} \sim 1$, the mass scales $M_{S}$ and $M_{S} / g_{s}^{2}$ coincide and calculability of the string theory is lost. The black holes are expected to dominate the dynamics above $M_{s}$.

However, neither black holes with masses less than 10.1 TeV nor string balls with masses less than $9.5 \mathrm{TeV}$ were 
seen at the LHC [58]. That is why, we assumed an absence of string-gravity corrections in our calculations.

\section{Conclusions}

Photon-induced exclusive processes $p p \rightarrow p \gamma \gamma p \rightarrow p X p$ are of great importance for high-energy physics. They provide one with unique precision measurements of the electroweak sector of the SM. They also allow us to study physics beyond the SM. For instance, (semi)exclusive $W W$ production by photon-photon interactions is very sensitive to quartic gauge anomalous couplings.

The dilepton production at the electroweak scale have been studied both at the Tevatron and LHC colliders. However, all previous experiments was done without a proton tag. Recently, the dimuon production in the process $p p \rightarrow p \gamma \gamma p^{*} \rightarrow p \mu^{+} \mu^{-} p^{*}$ have been studied with the CMS-TOTEM forward detector CT-PPS using measurements based on the integrated luminosity of $10 \mathrm{fb}^{-1}$ at $13 \mathrm{TeV}$ [59]. 12 events with $m_{\mu \mu}>110 \mathrm{GeV}$ matching forward detector kinematics were observed. This result is the first observation of proton-tagging $\gamma \gamma$ electroweak collisions.

In Ref. [47] a potential of the photon-induced dilepton final states at the LHC for a phenomenology of two models with the extra dimensions was investigated. The constraints both on the fundamental gravity scale $M_{D}$ in the ADD model and on the pair $\beta-m_{1}$ in the RS1 model (where $\beta=\kappa / \bar{M}_{5}$, $m_{1}$ is a mass of the lightest graviton), were derived [47].

In the present paper we have studied the photon-induced production of the muon pair at the LHC for $14 \mathrm{TeV}$ in the RSSC model with the warped extra dimension and small curvature [44-46]. For two acceptance regions, $0.0015<\xi<$ 0.5 and $0.1<\xi<0.5$, where $\xi$ is the proton energy fraction loss, the distributions in the muon transverse momenta $p_{t}$ are calculated as a function of the reduced fundamental gravity scale $\bar{M}_{5}$ and curvature parameter $\kappa$. It is shown that the deviation from the SM gets higher as $p_{t}$ grows. The obtained cross sections $\sigma\left(p_{t}>p_{t, \mathrm{~min}}\right)$ almost do not change in the region $0<p_{t, \min }<500 \mathrm{GeV}$ for the case $0.1<\xi<0.5$. It means that the high value of $\xi_{\text {min }}$ mimics the high value of $p_{t, \text { min }}$. The $95 \%$ L.C. discovery limits on $\bar{M}_{5}$ are obtained for the acceptance region $0.0015<\xi<0.5$ with $p_{t}>500 \mathrm{GeV}$ and for $0.1<\xi<0.5$ with $p_{t}>30 \mathrm{GeV}$. Note that the cut $|\eta|<2.4$ was imposed on the rapidities of the final muons.

Let us stress that our limits on $\bar{M}_{5}$ do not depend on the curvature parameter $\kappa$. In the RSSC model this fact also takes place for other processes, provided the inequality $\kappa \ll \bar{M}_{5}$ is satisfied [48]. Such a weak dependence on $\kappa$ for $\kappa \ll \bar{M}_{5}$ can be understood as follows. Consider a contribution of a gravity resonance with the mass $m_{0}=\sqrt{s \tau_{0}}$ to the sum $\mathcal{S}(s)$ (26). Its real part has two peaks with opposite signs which cancel each other. As for the imaginary part of this resonance, its height is proportional to $1 / \varepsilon_{0}$, where

$\varepsilon_{0}=\frac{\eta}{2}\left(\frac{\sqrt{s \tau_{0}}}{\bar{M}_{5}}\right)^{3}$,

while its width is equal to $2 \delta_{0}$, where

$\delta_{0}=\eta \frac{\kappa s \tau_{0}}{\bar{M}_{5}^{3}}$.

The total number of the graviton resonances which contribute to the differential cross section $d \sigma / d p_{t}^{2}$ is proportional to $N / \kappa$. As a result, we find that the gravity contribution to the differential cross section is proportional to

$s^{2}\left[\frac{1}{\bar{M}_{5}^{3} \sqrt{s} \varepsilon_{0}}\right]^{2} \delta_{0} N \sim \frac{1}{\bar{M}_{5}^{3} \sqrt{s}}$.

Thus, the smallness of the coupling constant $1 / \Lambda_{\pi}^{2}=\kappa / \bar{M}_{5}^{3}$ in (24) is compensated by a large number of gravitons $N=$ $\sqrt{s} / \kappa$ which give significant contribution to $\mathcal{S}(s)$.

It is in contrast to the RS1 model, in which $\kappa \sim \bar{M}_{5} \sim M_{\mathrm{Pl}}$. As a result, the RS1 discovery limits on $m_{1}$ for all processes, including photon-induced collisions at the LHC [47], depend on a chosen value of $\kappa$.

Open Access This article is distributed under the terms of the Creative Commons Attribution 4.0 International License (http://creativecomm ons.org/licenses/by/4.0/), which permits unrestricted use, distribution, and reproduction in any medium, provided you give appropriate credit to the original author(s) and the source, provide a link to the Creative Commons license, and indicate if changes were made. Funded by SCOAP ${ }^{3}$.

\section{Appendix A}

Symbols $C^{\alpha \beta \rho \sigma}$ and $D^{\alpha \beta \rho \sigma}$ in Eq. (19) are defined as follows

$$
\begin{aligned}
C^{\alpha \beta \rho \sigma}= & \eta^{\alpha \rho} \eta^{\beta \sigma}+\eta^{\alpha \sigma} \eta^{\beta \rho}-\eta^{\alpha \beta} \eta^{\rho \sigma}, \\
D^{\alpha \beta \rho \sigma}= & \eta^{\alpha \beta} k_{1}^{\sigma} k_{2}^{\rho}-\left(\eta^{\alpha \sigma} k_{1}^{\beta} k_{2}^{\rho}+\eta^{\alpha \rho} k_{1}^{\sigma} k_{2}^{\beta}-\eta^{\rho \sigma} k_{1}^{\alpha} k_{2}^{\beta}\right) \\
& -\left(\eta^{\beta \sigma} k_{1}^{\alpha} k_{2}^{\rho}+\eta^{\beta \rho} k_{1}^{\sigma} k_{2}^{\alpha}-\eta^{\rho \sigma} k_{1}^{\beta} k_{2}^{\alpha}\right) .
\end{aligned}
$$

\section{References}

1. L. Adamczyk et al., Technical Report. CERN-LHCC-2015-009, ATLAS-TDR-024 (2015)

2. M. Albrow et al., CMS-TOTEM, Technical Report. CERN-LHCC2014-021, TOTEM-TDR-003, CMS-TDR-13 (2014)

3. ATLAS Collaboration, Technical Report. CERN-LHCC-2011012, LHCC-I-020 (2011)

4. L. Adamczyk et al., Technical Report. ATLCOM-LUM-2011-006, CERN (2011)

5. G. Antchev et al. (TOTEM Collaboration), EPL 96, 21002 (2011)

6. G. Antchev et al. (TOTEM Collaboration), EPL 98, 31002 (2012)

7. G. Antchev et al., TOTEM Collaboration. EPL 101, 21002 (2013) 
8. M. Albrow et al. (FP420 R and D Collaboration), JINST 4, T10001 (2009)

9. M.G. Albrowa, T.D. Coughlin, J.R. Forshaw, Prog. Part. Nucl. Phys. 65, 149 (2010)

10. T. Aaltonen et al. (CDF Collaboration), Phys. Rev. Lett. 102, 242001 (2009)

11. T. Aaltonen et al. (CDF Collaboration), Phys. Rev. Lett. 102, 222002 (2009)

12. S. Chatrchyan et al. (CMS Collaboration), JHEP 1201, 052 (2012)

13. S. Chatrchyan et al. (CMS Collaboration), JHEP 1211, 080 (2012)

14. G. Aad et al. (ATLAS Collaboration), Phys. Lett. B 749, 242 (2015)

15. G. Aad et al. (ATLAS Collaboration), JHEP 08, 009 (2016)

16. K. Piotrzkowski, Phys. Rev. D 63, 071502(R) (2001)

17. V. Goncalves, M. Machado, Phys. Rev. D 75, 031502(R) (2007)

18. İ. Şahin, S.C. İnan, JHEP 09, 069 (2009)

19. S.C. İnan, Phys. Rev. D 81, 115002 (2010)

20. S. Atağ, A. Billur, JHEP 11, 060 (2010)

21. İ. Şahin, A.A. Billur, Phys. Rev. D 83, 035011 (2011)

22. İ. Şahin, M. Köksal, JHEP 11, 100 (2011)

23. S.C. İnan, A.A. Billur, Phys. Rev. D 84, 095002 (2011)

24. R.S. Gupta, Phys. Rev. D 85, 014006 (2012)

25. İ. Şahin, Phys. Rev. D 85, 033002 (2012)

26. B. Şahin, A.A. Billur, Phys. Rev. D 85, 074026 (2012)

27. L.N. Epele et al., Eur. Phys. J. Plus 127, 60 (2012)

28. İ. Şahin, B. Şahin, Phys. Rev. D 86, 115001 (2012)

29. A.A. Billur, Europhys. Lett. 101, 21001 (2013)

30. İ. Şahin et al., Phys. Rev. D 88, 095016 (2013)

31. H. Sun, C.X. Yue, Eur. Phys. J. C 74, 2823 (2014)

32. H. Sun, Nucl. Phys. B 886, 691 (2014)

33. İ. Şahin et al., Phys. Rev. D 91, 035017 (2015)

34. M. Köksal, S.C. İnan, Adv. High Energy Phys. 2014, 315826 (2014)

35. S. Fichet, JHEP 1704, 088 (2017)

36. C. Baldenegro, JHEP 1706, 142 (2017)
37. V. Budnev, I. Ginzburg, G. Meledin, V. Serbo, Phys. Rep. 15, 181 (1975)

38. G. Baur et al., Phys. Rep. 364, 359 (2002)

39. L. Randall, R. Sundrum, Phys. Rev. Lett. 83, 3370 (1999)

40. N. Arkani-Hamed, S. Dimopoulos, G. Dvali, Phys. Lett. B 429, 263 (1998)

41. N. Arkani-Hamed, S. Dimopoulos, G. Dvali, Phys. Rev. D 59, 086004 (1999)

42. I. Antoniadis, N. Arkani-Hamed, S. Dimopoulos, G. Dvali, Phys. Lett. B 436, 257 (1998)

43. A.V. Kisselev, Nucl. Phys. B 909, 218 (2016)

44. G.F. Giudice, T. Plehn, A. Strumia, Nucl. Phys. B 706, 455 (2005)

45. A.V. Kisselev, V.A. Petrov, Phys. Rev. D 71, 124032 (2005)

46. A.V. Kisselev, Phys. Rev. D 73, 024007 (2006)

47. S. Atağ, S.C. İnan, İ. Şahin, Phys. Rev. D 80, 075009 (2009)

48. A.V. Kisselev, JHEP 0809, 039 (2008)

49. A.V. Kisselev, JHEP 1304, 025 (2013)

50. G. Cowan, K. Cranmer, E. Gross, O. Vitells, Eur. Phys. J. C 71, 1554 (2011)

51. J. Polchinski, String Theory, vols. I and II (Cambridge University Press, Cambridge, 1998)

52. S. Dimopoulos, R. Emparan, Phys. Lett. B 526, 393 (2002)

53. T. Banks, W. Fischler, arXiv:hep-th/9906038

54. S. Dimopoulos, G. Landsberg, Phys. Rev. Lett. 87, 161602 (2001)

55. S.B. Giggings, eConf C010630, P328 (2001)

56. S.B. Giddings, S. Tomas, Phys. Rev. D 65, 056010 (2002)

57. G.F. Giudice, R. Rattazzi, J.D. Wells, Nucl. Phys. B 630, 293 (2002)

58. A.M. Sininyan et al., CMS Collaboration. Phys. Lett. B 774, 279 (2017)

59. A.M. Sininyan et al., CMS - TOTEM Collaboration. JHEP 07, 153 (2018) 